Research

Open Access

\title{
Using an expiratory resistor, arterial pulse pressure variations predict fluid responsiveness during spontaneous breathing: an experimental porcine study
}

\author{
Michael K Dahl, Simon T Vistisen, Jacob Koefoed-Nielsen and Anders Larsson
}

\author{
Anaesthesia and Intensive Care Medicine, North Denmark Region, Aalborg Hospital - Aarhus University Hospitals, Hobrovej 18-22, DK-9000 \\ Aalborg, Denmark \\ Corresponding author: Michael K Dahl, mida@rn.dk
}

Received: 23 Oct 2008 Revisions requested: 14 Jan 2009 Revisions received: 11 Feb 2009 Accepted: 20 Mar 2009 Published: 20 Mar 2009

Critical Care 2009, 13:R39 (doi:10.1186/cc7760)

This article is online at: http://ccforum.com/content/13/2/R39

(c) 2009 Dahl et al.; licensee BioMed Central Ltd.

This is an open access article distributed under the terms of the Creative Commons Attribution License (http://creativecommons.org/licenses/by/2.0), which permits unrestricted use, distribution, and reproduction in any medium, provided the original work is properly cited.

\begin{abstract}
Introduction Fluid responsiveness prediction is difficult in spontaneously breathing patients. Because the swings in intrathoracic pressure are minor during spontaneous breathing, dynamic parameters like pulse pressure variation (PPV) and systolic pressure variation (SPV) are usually small. We hypothesized that during spontaneous breathing, inspiratory and/or expiratory resistors could induce high arterial pressure variations at hypovolemia and low variations at normovolemia and hypervolemia. Furthermore, we hypothesized that SPV and PPV could predict fluid responsiveness under these conditions.

Methods Eight prone, anesthetized and spontaneously breathing pigs ( 20 to $25 \mathrm{~kg}$ ) were subjected to a sequence of $30 \%$ hypovolemia, normovolemia, and $20 \%$ and $40 \%$ hypervolemia. At each volemic level, the pigs breathed in a randomized order either through an inspiratory and/or an expiratory threshold resistor $\left(7.5 \mathrm{cmH}_{2} \mathrm{O}\right)$ or only through the tracheal tube without any resistor. Hemodynamic and respiratory variables were measured during the breathing modes. Fluid responsiveness was defined as a $15 \%$ increase in stroke volume $(\Delta S V)$ following fluid loading.
\end{abstract}

Results Stroke volume was significantly lower at hypovolemia compared with normovolemia, but no differences were found between normovolemia and $20 \%$ or $40 \%$ hypervolemia. Compared with breathing through no resistor, SPV was magnified by all resistors at hypovolemia whereas there were no changes at normovolemia and hypervolemia. PPV was magnified by the inspiratory resistor and the combined inspiratory and expiratory resistor. Regression analysis of SPV or PPV versus $\triangle$ SV showed the highest $R^{2}$ ( 0.83 for SPV and 0.52 for PPV) when the expiratory resistor was applied. The corresponding sensitivity and specificity for prediction of fluid responsiveness were $100 \%$ and $100 \%$, respectively, for SPV and $100 \%$ and $81 \%$, respectively, for PPV.

Conclusions Inspiratory and/or expiratory threshold resistors magnified SPV and PPV in spontaneously breathing pigs during hypovolemia. Using the expiratory resistor SPV and PPV predicted fluid responsiveness with good sensitivity and specificity.

\section{Introduction}

It may be difficult to assess whether a spontaneously breathing patient would hemodynamically benefit from intravenous fluid administration $[1,2]$. The oldest and most common procedure is observing whether blood pressure will drop by an upright tilt test - and the reverse to this procedure, leg raising, has recently been shown to accurately predict fluid responsiveness [3-5]. This procedure should be performed passively, however, and it is therefore not possible to perform with all beds or stretchers $[4,5]$. Static measures such as the central venous pressure or the pulmonary artery wedge pressure, if not extremely low, are not useful for assessment of fluid responsiveness [6-8]. A fluid challenge may tip patients with borderline cardiac insufficiency into an overt pulmonary edema, necessitating ventilatory support.

During controlled mechanical ventilation using relatively large tidal volumes with the patient deeply sedated and musclerelaxed, dynamic measures such as pulse pressure variation (PPV) and systolic pressure variation (SPV) predict fluid

PPV: pulse pressure variation; SPV: systolic pressure variation; SV: stroke volume. 
responsiveness well [8-10]. These variations are caused by tidal changes in the intrathoracic pressure induced by positive pressure ventilation. During spontaneous breathing the changes in intrathoracic pressures are minimal and often the normal increase in arterial pressure during expiration is difficult to discern [11]. In pathological situations where the left heart filling is hampered during inspiration, such as cardiac tamponade, or when the right heart filling is reduced during expiration by high intrathoracic pressure, for example at acute exacerbation of chronic obstructive lung disease or asthma, however, the normal respiratory variations in arterial pressure may be enhanced, creating pulsus paradoxus [11,12]. In addition, pulsus paradoxus has been reported as a sign of severe hemorrhagic shock [12].

We hypothesized that a low level of expiratory resistance reducing right heart filling and, some beats later (during the inspiratory phase), reducing the left ventricular stroke volume (SV) - or an inspiratory resistance - enhancing the right heart filling and, some beats after, enhancing the left ventricular SV - could induce high arterial pressure variations at hypovolemia and low arterial pressure variations at normovolemia and hypervolemia. The SPV or PPV might therefore predict fluid responsiveness during spontaneous breathing when expiratory and/or inspiratory resistances are used. In addition, we hypothesized - because an expiratory resistance would theoretically give similar changes as repeated short Valsalva maneuvers (that is, initial augmentation of the arterial pressure followed by a depression) - that tidal changes in arterial pressure caused by an expiratory resistor might give similar or better information about fluid responsiveness than an inspiratory resistor or an inspiratory/expiratory resistor.

The aim of this study was to test in a porcine experimental model whether the SPV and the PPV would be magnified by an expiratory resistor, an inspiratory resistor or a combined inspiratory/expiratory resistor during hypovolemia, normovolemia and hypervolemia, and to test whether the SPV or PPV when using an expiratory resistor would predict the hemodynamic effect of subsequent fluid loading.

\section{Materials and methods}

The study was approved by the national animal ethics committee, and the National Institutes of Health principles of laboratory care were followed. Eight pigs, weighing 25 to $30 \mathrm{~kg}$, were premedicated with apazerone $80 \mathrm{mg}$ intramuscularly and midazolam $10 \mathrm{mg}$ intramuscularly. Anesthesia was induced by remifentanil $1 \mu \mathrm{g} / \mathrm{kg}$ intravenously and propofol $3 \mathrm{mg} / \mathrm{kg}$ intravenously. A tracheotomy was performed and the trachea was intubated with a Portex 9.0 ID tube (Smiths Medical, London, UK). The lungs were ventilated by a Servo $900 \mathrm{C}$ ventilator (Siemens-Elema, Solna, Sweden) with volume control, tidal volume of $8 \mathrm{ml} / \mathrm{kg}$, positive end-expiratory pressure of 5 $\mathrm{cmH}_{2} \mathrm{O}$ and a fraction of inspired oxygen of 1.0. The inspiratory time was $35 \%$, the end-inspiratory pause time was $10 \%$ and the ventilatory rate was adjusted to achieve an arterial $\mathrm{pH}$ of approximately 7.4. Anesthesia was maintained with ketamine $10 \mathrm{mg} / \mathrm{kg} / \mathrm{hour}$, remifentanil $0.5 \mu \mathrm{g} / \mathrm{kg} / \mathrm{hour}$ and propofol $10 \mathrm{mg} / \mathrm{kg} /$ hour. Ringer's acetate $20 \mathrm{ml} / \mathrm{kg}$ was infused during the instrumentation phase. In one animal, a bolus of Ringer's acetate $10 \mathrm{ml} / \mathrm{kg}$ was administered to stabilize circulation before the main experiment. Monitoring with electrocardiography and pulse oximetry (placed on the tail) was initiated.

Catheters were placed in the right carotid artery, in a femoral artery, and in the right internal jugular vein for sampling of blood gases, monitoring of intravascular pressures and obtaining the pulse contour cardiac output. A pulmonary artery catheter (Swan-Ganz $\mathrm{CCO}$ mbo $\mathrm{CCO} / \mathrm{SvO}_{2} 7.5 \mathrm{Fr}$; Edwards Lifescience, Irvine, CA, USA) was placed via the right external jugular vein to monitor the pulmonary artery and central venous pressures. A suprapubic urinary catheter was inserted for monitoring diuresis.

An air-filled $6 \mathrm{Fr}$ catheter was inserted in the tracheal tube with the end-hole $1 \mathrm{~cm}$ below the distal opening of the tracheal tube for airway pressure monitoring. The distal esophageal pressure was measured via a latex balloon catheter (Viasys Healthcare, Hochberg, Germany) and an adequate position was ensured as previously described [13]. The tracheal and esophageal catheters were connected to transducers (Edwards Lifesciences) and the signals were transferred to a monitor (S/5 Avance Carestation; GE Healthcare, Chalfont St Giles, UK).

The pulse contour cardiac output was obtained through the catheter (Pulsiocath, 4 Fr, $16 \mathrm{~cm}$; Pulsion Medical Systems, Munich, Germany) placed in a femoral artery connected to the $\mathrm{PiCCO}$ monitor (Pulsion Medical Systems). The pulse contour cardiac output measurement was calibrated in triple with the transpulmonary arterial thermodilution technique using cold saline injectate $(3 \times 10 \mathrm{ml})$ immediately after induction of anesthesia and before each measurement sequence. In addition, the intrathoracic blood volume and PPV were obtained from the PiCCO device.

During the entire study period, electrocardiography, the cardiac output, blood pressures, the heart rate, and the airway and esophageal pressures were recorded continuously for later analyses. Blood gases were sampled from the right carotid and the pulmonary artery and were analyzed by an ABL 710 (Radiometer, Copenhagen, Denmark).

\section{Experimental protocol}

The outline of the experiment is shown in Figure 1. After instrumentation, the animal was placed prone and an interval of 20 minutes was allowed before spontaneous breathing was attempted; the ventilatory rate was reduced to one-half, the triggering level of the ventilator was set at $-1 \mathrm{cmH}_{2} \mathrm{O}$, the remifentanil infusion was stopped, and the ketamine and pro- 
Figure 1

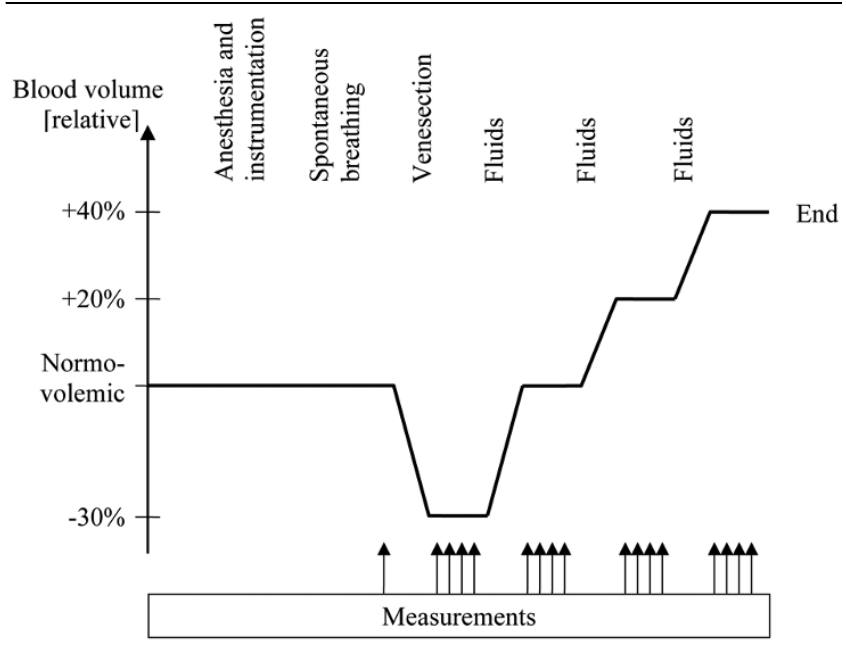

Outline of the experiment. The experimental procedure. Venesection, venesection of $30 \%$ of the estimated blood volume. Fluids, intravenous infusion of a starch solution of first $30 \%$ and then $20 \%$ of the estimated blood volume. Measurements, measurements of hemodynamic and respiratory variables. Tests were performed with the different resistors in a randomized order (see text). End, end of the experiment.

pofol infusions were halved and then adjusted to maintain adequate anesthesia (no movement and no reaction to painful stimulation of the anterior toes). When spontaneous breathing attempts began (the animal started to initiate breaths by triggering the ventilator), the ventilator was set to low-level pressure support $\left(2 \mathrm{cmH}_{2} \mathrm{O}\right.$ above the positive end-expiratory pressure). After about 2 minutes, the animal was connected to a spontaneous breathing device consisting of a Y-piece with inspiratory and expiratory valves and an anesthesia balloon with a valve regulating the oxygen flow from a flowmeter connected to a central pressurized oxygen source. The balloon was attached proximal to the inspiratory valve and the oxygen flow was regulated manually, keeping the balloon slightly expanded but still flaccid. In a bench test, the valves and the $\mathrm{Y}$-piece generated $<1 \mathrm{cmH}_{2} \mathrm{O}$ resistance to inspiratory and expiratory flow.

In the main experiment, when testing the effect of an expiratory resistance, the expiratory valve was replaced with a 7.5 $\mathrm{CmH}_{2} \mathrm{O}$ threshold resistor (CPAP; Philips Respironics, Herrshing, Germany); when the effect of an inspiratory resistance was tested, the inspiratory valve was replaced with a 7.5 $\mathrm{CmH}_{2} \mathrm{O}$ threshold resistor (CPAP; Philips Respironics); and when the effect of both expiratory and inspiratory resistances was tested, both resistors were connected as described above. In a bench test before the experiment, with the connectors used, the inspiratory resistor gave a resistance of 8.5 $\mathrm{cmH}_{2} \mathrm{O}$ and the expiratory resistor gave a resistance of 7.5 $\mathrm{cmH}_{2} \mathrm{O}$.
Baseline data were obtained during spontaneous breathing without a resistor. Thereafter, the main experiment was initiated. Measurements were performed when the animals breathed without a resistor, with the expiratory resistor, with the inspiratory resistor and with the inspiratory/expiratory resistor at four volemic levels: 30\% hypovolemia, normovolemia, and $20 \%$ and $40 \%$ hypervolemia. The order of breathing modes was randomized by computer randomization. Hypovolemia was achieved by venesection of $30 \%$ of the estimated blood volume, normovolemia was achieved by replacing the depleted blood with a starch solution (Voluven; Fresenius Kabi, Uppsala, Sweden), and 20\% and 40\% hypervolemia were achieved by infusion of corresponding volumes of the starch solution.

The blood volume was estimated as $179 \times$ body weight $^{(0.73)}$, which is about $8 \%$ of the body weight [14]. The sequence of intravascular volume levels was always hypovolemia, normovolemia, and $20 \%$ and $40 \%$ hypervolemia. Each infusion or blood removal was performed over 5 to 10 minutes. During these procedures the animal breathed with pressure support using the settings as described above. This was followed by a 5-minute stabilization period with spontaneous breathing before a new measurement sequence was performed. Electrocardiography, the cardiac output, systolic arterial blood pressures, the heart rate, the pulmonary artery wedge pressure, the central venous pressure, the intrathoracic blood volume, and the SPV and PPV were registered 3 minutes after the resistor change. Blood gases were sampled, and the airway and esophageal pressures were obtained for calculation of the transpulmonary pressure and respiratory intrathoracic pressure variations.

After the experiment, the animal was killed by an overdose of thiopental and potassium chloride intravenously.

\section{Calculations}

Fluid responsiveness was defined as an increase in the SV of $15 \%$ after fluid loading.

Before the study, we decided to manually calculate the PPV and the SPV from the pressure tracings, because we have previously found a significant variation in the PiCCO monitor's stated SPV and PPV values during controlled ventilation of pigs [15]. We had problems with measuring the PPV correctly, however, and therefore the PPV was obtained automatically from the PiCCO device. The SPV was calculated over six respiratory cycles as previously described by Michard and colleagues [16].

The SV was obtained as the ratio of cardiac output/heart rate.

Airway pressure variations were calculated as the mean values for six respiratory cycles of maximal airway pressure (expiration) minus minimum airway pressure (inspiration). The same 
calculations were carried out regarding the pleural (esophageal) pressure. The transpulmonary pressure was obtained as the airway pressure minus esophageal pressure at similar time points, and the variations were registered simultaneously with the airway pressure.

\section{Statistical analysis}

The statistical analyses were performed using the SigmaStat 3.5 program (Systat Inc., Point Richmond, CA, USA). Results are presented as the mean and standard deviation, if not otherwise indicated. $P<0.05$ was considered significant. Normal distribution of the data was checked with the KolmogorovSmirnov test.

The overall changes in cardiac output, SV, central venous pressure and intrathoracic blood volume between the different volemic levels for no resistor were analyzed by one-way analysis of variance and the Tukey test. The overall changes in PPV and SPV between the different volemic levels with the different resistors in place were analyzed by two-way analysis of variance and the Tukey test. The differences in hemodynamics and in respiratory pressures caused by the different resistors at $30 \%$ hypovolemia were analyzed by one-way analysis of variance and the Tukey test. The relation between the SV and the
SPV or PPV was analyzed by linear regression, and the sensitivity and specificity were calculated by standard formulas after inspection of the receiver operating characteristic curves (SigmaPlot 11.0; Systat Inc.).

\section{Results Hemodynamics without a resistor}

The cardiac output, the SV, the central venous pressure and the intrathoracic blood volume were significantly lower during hypovolemia than during normovolemia, whereas there were minor or insignificant changes between the other volemic steps (Table 1). The SPV was similar at all volemic levels, whereas the PPV was significantly higher at $-30 \%$ hypovolemia (Table 1).

\section{Effects of resistors on airway and esophageal pressures} The airway and esophageal pressure swings were generally higher with resistors than without a resistor (Table 2). The transpulmonary pressure swings were somewhat higher with the inspiratory/expiratory resistor compared with no resistor, indicating larger tidal volumes.

Table 1

Central hemodynamics and arterial pressure variations at the four volemic levels

\begin{tabular}{|c|c|c|c|c|}
\hline & $-30 \%$ hypovolemia & $0 \%$ normovolemia & $+20 \%$ hypervolemia & $+40 \%$ hypervolemia \\
\hline \multicolumn{5}{|l|}{ No resistor } \\
\hline Cardiac output (I/min) & $3.2 \pm 0.7$ & $7.5 \pm 1.6^{*}$ & $7.9 \pm 2.0$ & $7.7 \pm 2.2$ \\
\hline Stroke volume (ml) & $24 \pm 5$ & $65 \pm 11^{*}$ & $63 \pm 10$ & $62 \pm 10$ \\
\hline Central venous pressure $(\mathrm{mmHg})$ & $0 \pm 2$ & $6 \pm 2^{*}$ & $7 \pm 2^{*}$ & $8 \pm 2^{*}$ \\
\hline Intrathoracic blood volume (ml) & $485 \pm 88$ & $814 \pm 177^{*}$ & $849 \pm 156$ & $924 \pm 213$ \\
\hline Central venous oxygen saturation & $0.89 \pm 0.05$ & $0.99 \pm 0.04^{*}$ & $1 \pm 0.02$ & $0.98 \pm 0.04$ \\
\hline Lactate $(\mathrm{mmol} / \mathrm{l})$ & $1.2 \pm 1.3$ & $2.4 \pm 1.8$ & $1.9 \pm 1.2$ & $1.2 \pm 0.8$ \\
\hline Base excess $(\mathrm{mmol} / \mathrm{l})$ & $4.1 \pm 1.5$ & $2.2 \pm 1.7$ & $2.2 \pm 1.6$ & $3.0 \pm 1.9$ \\
\hline \multicolumn{5}{|l|}{ Systolic pressure variation } \\
\hline No resistor (\%) & $5 \pm 2$ & $3 \pm 2$ & $2 \pm 1$ & $2 \pm 1$ \\
\hline Inspiratory resistor (\%) & $10 \pm 5^{\dagger}$ & $4 \pm 2^{*}$ & $5 \pm 2$ & $4 \pm 2$ \\
\hline Expiratory resistor (\%) & $11 \pm 2^{\dagger}$ & $4 \pm 2^{*}$ & $4 \pm 1$ & $3 \pm 2$ \\
\hline Inspiratory/expiratory resistor (\%) & $13 \pm 5^{+}$ & $5 \pm 3^{*}$ & $5 \pm 2$ & $4 \pm 2$ \\
\hline \multicolumn{5}{|l|}{ Pulse pressure variation } \\
\hline No resistor (\%) & $17 \pm 5$ & $12 \pm 2^{\star}$ & $12 \pm 4$ & $12 \pm 1$ \\
\hline Inspiratory resistor (\%) & $25 \pm 6^{\dagger}$ & $16 \pm 4^{\star}+$ & $16 \pm 6^{+}$ & $15 \pm 5^{\dagger}$ \\
\hline Expiratory resistor (\%) & $25 \pm 6$ & $13 \pm 6^{*}$ & $12 \pm 3$ & $11 \pm 3$ \\
\hline Inspiratory/expiratory resistor (\%) & $26 \pm 7^{+}$ & $14 \pm 6^{\star}+$ & $14 \pm 5^{\dagger}$ & $13 \pm 6^{\dagger}$ \\
\hline
\end{tabular}

Data presented as the mean \pm standard deviation. ${ }^{\star} P<0.05$ compared with the previous volemic level. ${ }^{t} P<0.05$ compared with no resistor at the same volemic level. 
Table 2

\begin{tabular}{|c|c|c|c|c|}
\hline & No resistor & Inspiratory resistor & Expiratory resistor & Inspiratory/expiratory resistor \\
\hline \multicolumn{5}{|l|}{ Airway pressure (AP) } \\
\hline Inspiratory $\left(\mathrm{cmH}_{2} \mathrm{O}\right)$ & $-1 \pm 4$ & $-7 \pm 2^{\star}$ & $-3 \pm 4$ & $-5 \pm 2^{*}$ \\
\hline Expiratory $\left(\mathrm{cmH}_{2} \mathrm{O}\right)$ & $3 \pm 5$ & $1 \pm 2$ & $5 \pm 2$ & $5 \pm 2$ \\
\hline$\Delta \mathrm{AP}\left(\mathrm{cmH}_{2} \mathrm{O}\right)$ & $4 \pm 1$ & $8 \pm 1^{*}$ & $8 \pm 2^{\star}$ & $11 \pm 4^{\star}$ \\
\hline \multicolumn{5}{|l|}{ Esophageal pressure (EP) } \\
\hline Inspiratory $\left(\mathrm{cmH}_{2} \mathrm{O}\right)$ & $-4 \pm 2$ & $-9 \pm 3^{*}$ & $-6 \pm 3$ & $-8 \pm 2^{*}$ \\
\hline Expiratory $\left(\mathrm{cmH}_{2} \mathrm{O}\right)$ & $-2 \pm 1$ & $-3 \pm 3$ & $-1 \pm 2$ & $-2 \pm 3$ \\
\hline$\Delta \mathrm{EP}\left(\mathrm{cmH}_{2} \mathrm{O}\right)$ & $3 \pm 1$ & $6 \pm 1^{*}$ & $5 \pm 2^{*}$ & $6 \pm 2^{*}$ \\
\hline \multicolumn{5}{|l|}{ Transpulmonary pressure (TP) } \\
\hline Inspiratory $\left(\mathrm{cmH}_{2} \mathrm{O}\right)$ & $3 \pm 4$ & $2 \pm 4$ & $4 \pm 2$ & $3 \pm 4$ \\
\hline Expiratory $\left(\mathrm{cmH}_{2} \mathrm{O}\right)$ & $5 \pm 5$ & $5 \pm 4$ & $6 \pm 1$ & $7 \pm 3$ \\
\hline$\Delta \mathrm{TP}\left(\mathrm{cmH}_{2} \mathrm{O}\right)$ & $1 \pm 2$ & $3 \pm 1$ & $2 \pm 1$ & $4 \pm 3^{*}$ \\
\hline Heart rate (/min) & $130 \pm 21$ & $133 \pm 12$ & $138 \pm 18$ & $137 \pm 23$ \\
\hline Cardiac output (I/min) & $3.2 \pm 0.7$ & $3.3 \pm 0.4$ & $3.3 \pm 0.5$ & $3.2 \pm 0.5$ \\
\hline Stroke volume (ml) & $25 \pm 5$ & $25 \pm 4$ & $24 \pm 4$ & $24 \pm 5$ \\
\hline PAWP during inspiration $(\mathrm{mmHg})$ & $-2 \pm 5$ & $-7 \pm 4$ & $-3 \pm 4$ & $-5 \pm 3$ \\
\hline PAWP during expiration $(\mathrm{mmHg})$ & $4 \pm 3$ & $6 \pm 2$ & $8 \pm 2^{*}$ & $7 \pm 2$ \\
\hline Mean arterial pressure $(\mathrm{mmHg})$ & $55 \pm 6$ & $59 \pm 5$ & $60 \pm 7$ & $59 \pm 5$ \\
\hline Central venous pressure $(\mathrm{mmHg})$ & $0 \pm 2$ & $-1 \pm 3$ & $1 \pm 3$ & $1 \pm 3$ \\
\hline
\end{tabular}

Data presented as the mean \pm standard deviation. ${ }^{\star} P<0.05$ compared with no resistor. PAWP, pulmonary artery wedge pressure.

Hemodynamic consequences at each volemic level of applying the resistors

At each volemic level, the cardiac output, the SV, the mean arterial pressure and the heart rate did not change when applying the resistors, whereas the swings in pulmonary artery wedge pressure were slightly related to the swings in airway pressure $\left(R^{2}=0.12\right)$ (Table 2$)$.

At 30\% hypovolemia, as compared with no resistor, the SPV was magnified by all resistors, whereas no changes were found at normovolemia and at $20 \%$ and $40 \%$ hypervolemia.
The PPV was magnified by the inspiratory resistor and the inspiratory/expiratory resistor (Table 1).

\section{Correlations between changes in stroke volume and systolic or pulse pressure variation using the different resistors}

The regression analyses between the change in SV and the SPV or PPV using the different resistors are presented in Table 3. The $R^{2}$ value was generally higher when the expiratory resistor was applied with the highest correlation $\left(R^{2}=0.83\right)$ for the SPV.

Table 3

Correlation of systolic pressure variation and pulse pressure variation versus the change in stroke volume

\begin{tabular}{lll}
\hline & Systolic pressure variation & Pulse pressure variation \\
\hline No resistor & 0.37 & 0.37 \\
Inspiratory resistor & 0.45 & 0.36 \\
Expiratory resistor & 0.83 & 0.52 \\
Inspiratory/expiratory resistor & 0.50 & 0.31
\end{tabular}

Data presented as $R^{2}$ values obtained by linear regression for systolic pressure variation or pulse pressure variation versus the increase in stroke volume by a subsequent fluid loading with no resistor and with the inspiratory, expiratory, and inspiratory/expiratory resistors. 


\section{Performances of systolic pressure and pulse pressure variations for each resistor}

Using a 15\% increase in SV as the definition of fluid responsiveness, the sensitivity and specificity for SPV and PPV were as shown in Table 4. The highest sensitivity was found for the expiratory resistor. The SPV gave sensitivity and specificity of $100 \%$ for a SPV cutoff value of $7 \%$ with the expiratory resistor, and sensitivity and specificity of $63 \%$ and $94 \%$, respectively, for a cutoff value of $4 \%$ without a resistor (Figures 2 and 3 ). Corresponding values for the PPV were sensitivity and specificity of $100 \%$ and $81 \%$, respectively, and sensitivity and specificity of $88 \%$ and $69 \%$, respectively, for PPV cutoff values of $16 \%$ and $13 \%$, respectively (Figures 2 and 3 ).

\section{Central venous oxygen saturation, lactate and blood gases}

The central venous oxygen saturation increased from normovolemia, whereas the partial arterial tension of oxygen and the partial arterial tension of carbon dioxide (data not shown) as well as the base excess and lactate were stable during the experiment, with no significant changes between the volemic levels or respiratory modes.

\section{Discussion}

We have shown in this exploratory study in spontaneously breathing pigs that inspiratory and/or expiratory threshold resistors magnified arterial pressure variations markedly during hypovolemia, whereas changes in arterial pressure variations were minor during normovolemia and hypervolemia; that the expiratory resistor gave a better relation between the SPV or PPV and the change in SV by subsequent fluid loading than the inspiratory resistor or the inspiratory/expiratory resistor; and that the SPV and PPV using the expiratory resistor predicted fluid responsiveness with good sensitivity and specificity.

We manipulated the intrathoracic pressure to magnify the normal swings in arterial pressure. This concept has long been used clinically during controlled mechanical ventilation [8-10]. The ventilator-induced cyclic changes in intrathoracic pressure produce significant arterial pressure variations if the circulation is fluid responsive. The tidal volume, however, has to be above $8 \mathrm{ml} / \mathrm{kg}$ predicted body weight [17], which is higher than recommended in critically ill, ventilated patients [18]. Furthermore, the patient should have normal right heart function, no atrial fibrillation, and no spontaneous breathing activity [8-

Figure 2
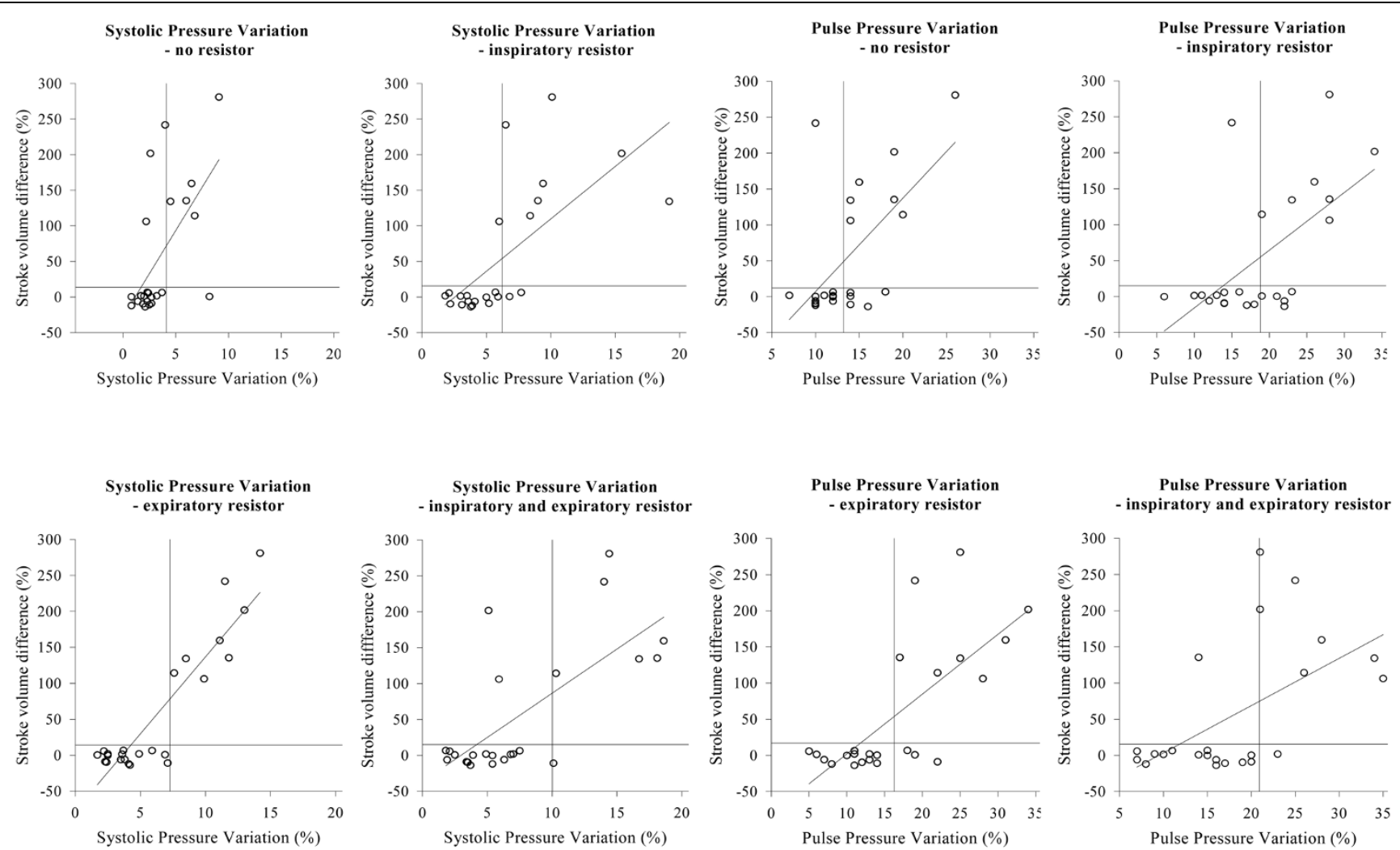

Linear regression for systolic pressure variation and pulse pressure variation. Systolic pressure variation and pulse pressure variation before fluid administration versus the change in stroke volume following fluid loading without and with the expiratory resistor. Regression lines are indicated. All measurement points are used in the regression analyses. Horizontal lines, relevant change in stroke volume (15\%); vertical lines, cutoff values used. 
Figure 3
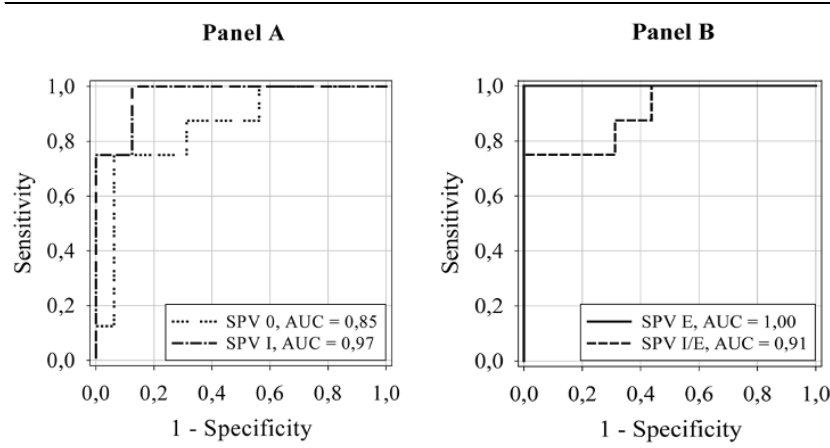

Panel C
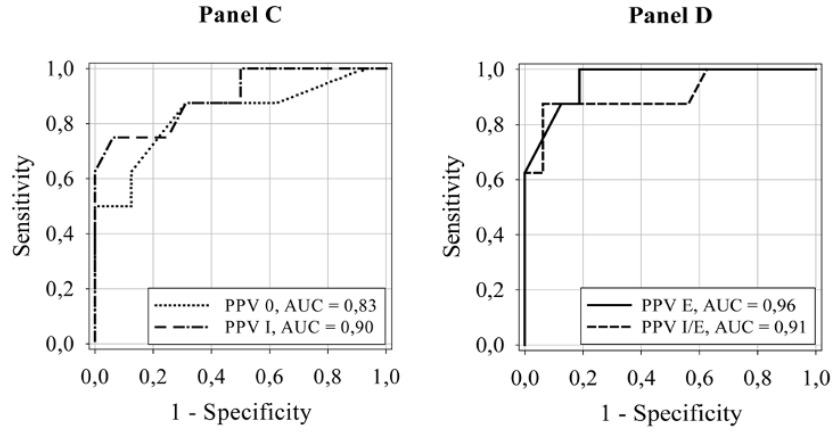

Receiver operating characteristic curves for systolic pressure variation and pulse pressure variation. Receiver operating characteristic curves for (a), (b) systolic pressure variation and (c), (d) pulse pressure variation, with the four different respiratory interventions, for predicting a $15 \%$ increase in stroke volume by subsequent fluid loading. SPV 0 , systolic pressure variation with no resistor; SPV I, systolic pressure variation with the inspiratory resistor; SPV E, systolic pressure variation with the expiratory resistor; SPV I/E, systolic pressure variation with the combined inspiratory and expiratory resistor; PPV 0 , pulse pressure variation with no resistor; PPV I, pulse pressure variation with the inspiratory resistor; PPV E, pulse pressure variation with the expiratory resistor; PPV I/E, pulse pressure variation with the combined inspiratory and expiratory resistor; AUC, area under the curve.

10]. Indeed, if the patient is breathing in a spontaneous ventilator mode, the arterial pressure variations will not give any information about fluid responsiveness [19].

In spontaneously breathing, hemodynamically unstable patients, Soubrier and colleagues found a sensitivity and specificity for predicting the effect of a subsequent fluid administration of $63 \%$ and $92 \%$, respectively, for the PPV, and a sensitivity and specificity of $47 \%$ and $92 \%$, respectively, for the SPV - as discussed in the accompanying editorial [20] agreeing well with our results without resistors. Our study therefore confirms that arterial pressure variations during normal spontaneous breathing are not useful for fluid responsiveness prediction, mainly because of low sensitivity. Soubrier and colleagues also investigated whether a forceful inspiration and expiration (with no resistance) would improve the ability of the SPV and the PPV to predict fluid responsiveness [21]. The sensitivity was even lower, however, with this maneuver [21].
Indeed, we found a somewhat lower sensitivity with the expiratory/inspiratory resistor for SPV than with the other resistors.

In the editorial to the paper by Soubrier and colleagues, de Backer and Pinsky discussed whether manipulation of the intrathoracic pressure by a Valsalva maneuver - that is, a forceful expiration against a resistance - could be used to generate arterial pressure variations that could predict fluid responsiveness [20]. In fact, this has now been shown in a very recent study by Garcia and colleagues [22]. A Valsalva maneuver causes an immediate increase in cardiac output by squeezing blood from the pulmonary circulation to the left heart, but this is very quickly followed by a marked reduction in cardiac output due to reduced right heart filling [23]. As the Valsalva maneuver may induce a pronounced drop in blood pressure during hypovolemia, it may be difficult to perform in a patient distressed by circulatory compromise or pain - and the maneuver may induce changes in the heart rate. Moreover, the Valsalva maneuver may generate quite different intrathoracic pressures dependent on the patient's effort.

On the other hand, breathing against an expiratory resistance could be considered to give short, intermittent Valsalva maneuvers. This will cyclically reduce right heart filling and induce variations in arterial blood pressure that theoretically would be more pronounced when the circulation is fluid responsive. Indeed, in our study when using the expiratory resistor, the SPV was markedly enhanced during hypovolemia and became normalized during normovolemia; in addition, the SPV and the PPV could be used to predict fluid responsiveness. The minor difference in performance between the PPV and the SPV in our study is probably due to differences in obtaining these variables. The PPV was obtained from the PiCCO device and the SPV was obtained manually from the pressure tracings (see Calculations).

The inspiratory resistor and the inspiratory/expiratory resistor did also magnify the arterial pressure variations. Both of these resistors, however, gave inferior precision for fluid responsiveness prediction compared with the expiratory resistor. An explanation could be the different changes in intrathoracic pressures induced by the resistors; the expiratory resistor mainly increases the intrathoracic pressure during expiration, whereas the inspiratory resistor decreases the intrathoracic pressure during inspiration (Table 2). This decrease in the inspiratory intrathoracic pressure decreases left heart filling by reducing the pressure difference between the pulmonary vessels and the left atrium (as reflected in the markedly negative inspiratory pulmonary artery wedge pressure; Table 2), but simultaneously it improves right heart filling and thus, some beats afterwards, improves the left heart filling and the SV. Because of anatomical reasons the caval veins should be more affected by the pleural pressure than by the airway or the transpulmonary pressures, and thus the right heart filling should be dependent on the difference between the vein pres- 
Table 4

Sensitivity, specificity, positive and negative predictive values for the pressure variations with different respiratory interventions

\begin{tabular}{|c|c|c|c|c|}
\hline & Sensitivity (\%) & Specificity (\%) & Positive predictive value (\%) & Negative predictive value (\%) \\
\hline \multicolumn{5}{|l|}{ Systolic pressure variation } \\
\hline No resistor & 63 & 94 & 83 & 83 \\
\hline Inspiratory resistor & 88 & 88 & 78 & 93 \\
\hline Expiratory resistor & 100 & 100 & 100 & 100 \\
\hline Inspiratory/expiratory resistor & 75 & 94 & 86 & 88 \\
\hline \multicolumn{5}{|l|}{ Pulse pressure variation } \\
\hline No resistor & 88 & 69 & 58 & 92 \\
\hline Inspiratory resistor & 88 & 69 & 58 & 92 \\
\hline Expiratory resistor & 100 & 81 & 73 & 100 \\
\hline Inspiratory/expiratory resistor & 88 & 94 & 88 & 94 \\
\hline
\end{tabular}

sure and the pleural (esophageal) pressure. In fact, the inspiratory resistor reduced the inspiratory esophageal pressure and theoretically improved the right heart filling, whereas the expiratory device increased the expiratory esophageal pressure and theoretically reduced the right heart filling. The inspiratory/expiratory device had a combined effect.

The difference between the inspiratory and inspiratory/expiratory resistors could therefore be explained by the Frank-Starling heart function curve. With an expiratory resistor the filling becomes lower, causing the heart function to work on the steeper left part of the curve; whereas an inspiratory resistor improves filling, causing the heart function to work on the right less steep part of the curve. This would make the pressure variations with the expiratory resistor somewhat higher than with the inspiratory resistor, and the signal would be more pronounced. According to this reasoning, the inspiratory/expiratory resistor - making the heart work on a wider part of the Frank-Starling curve - would give highest pressure variations, agreeing with our result.

Inspiratory resistors have been found to improve cardiac output in experimental settings of hypovolemia [24,25]. We could not confirm this finding. The resistance level used in our study, however, was less than in the studies investigating the effect on cardiac output by inspiratory threshold resistors [24,25].

The use of an expiratory resistor connected to a nose-mouth mask is feasible in the clinic. It is used commonly for breathing physiotherapy in patients in the intensive care unit and in patients before and after surgery [26].

Our study has several limitations and caution should therefore be taken when translating the results to patients. First, we studied a limited number of young healthy animals with normal heart function and with no arrhythmias. Second, because we did not a priori know the effect on the arterial pressure variations by hypovolemia and volume challenges, both the hypovolemic level and the volume challenges were substantial and, furthermore, two different volume challenges were used. Third, the level of expiratory resistance used might not be optimal in patients. We chose these resistors because 5 to $10 \mathrm{cmH}_{2} \mathrm{O}$ is commonly used as expiratory impedance clinically (for example, for positive end-expiratory pressure or continuous positive airway pressure) and are accepted by most patients. Fourth, some values used in the receiver operating characteristic and linear regression analyses were dependent, making these analyses less strong.

\section{Conclusions}

The present exploratory animal study shows that arterial pressure variations predict fluid responsiveness during spontaneous breathing with an expiratory resistor.

\section{Key messages}

- Using an expiratory resistor, fluid responsiveness can be predicted by assessment of arterial pressure variations during spontaneous breathing.

\section{Competing interests}

The authors declare that they have no competing interests.

\section{Authors' contributions}

MKD and AL participated in the design, laboratory work, data analyses and writing of the manuscript. STV, JK-N participated in the design, the laboratory work and in the finalizing of the manuscript.

\section{References}

1. Magder S: Predicting volume responsiveness in spontaneously breathing patients: still a challenging problem. Crit Care 2006, 10:165. 
2. Coudray A, Romand J-A, Treggiari M, Bendjelid K: Fluid responsiveness in spontaneously breathing patients: a review of indexes used in intensive care. Crit Care Med 2005, 33:2757-2762.

3. Knopp R, Claypool R, Leonardi D: Use of the tilt test in measuring acute blood loss. Ann Emerg Med 1980, 9:72-75.

4. Monnet X, Rienzo M, Osman D, Anguel N, Richard C, Pinsky MR, Teboul JL: Passive leg raising predicts fluid responsiveness in the critically ill. Crit Care Med 2006, 34:1402-1407.

5. Teboul J-L, Monnet X: Prediction of volume responsiveness in critically ill patients with spontaneous breathing activity. Curr Opin Crit Care 2008, 14:334-339.

6. Marik PE, Baram M, Vahid B: Does central venous pressure predict fluid responsiveness? A systematic review of the literature and the tale of seven mares. Chest 2008, 134:172-178.

7. Michard F, Teboul JL: Predicting fluid responsiveness in ICU patients. A critical analysis of the evidence. Chest 2002, 121:2000-2008.

8. Bendjelid K, Romand JA: Fluid responsiveness in mechanically ventilated patients: a review of indices used in intensive care. Intensive Care Med 2003, 29:352-360.

9. Michard F: Changes in arterial pressure during mechanical ventilation. Anesthesiology 2005, 103:419-428.

10. Perel A: Assessing fluid responsiveness by the systolic pressure variation in mechanically ventilated patients. Systolic pressure variation as a guide to fluid therapy in patients with sepsis-induced hypotension. Anesthesiology 1998, 89:1309-1310.

11. Dornhorst AC, Howard P, Leathart GL: Pulsus paradoxus. Lancet 1952, 1:746-748.

12. Cohn JN, Pinkerson AL, Tristani FE: Mechanism of pulsus paradoxus in clinical shock. J Clin Invest 1967, 46:1744-1755.

13. Ingimarsson J, Thorsteinsson A, Larsson A, Werner O: Lung and chest wall mechanics in anesthetized children. Am J Respir Crit Care Med 2000, 162:412-417.

14. von Englehardt W: Swine cardiovascular physiology: a review. In Swine in Biomedical Research Edited by: Bustad LK, Mclellan RO. Seattle, WA: Frayn; 1966:307-329.

15. Lambert $P$, Sloth E, Smith B, Hansen LK, Koefoed-Nielsen J, Tønnesen $E$, Larsson A: Does a positive end-expiratory pressureinduced reduction in troke volume indicate preload responsiveness? An experimental study. Acta Anaesthesiol Scand 2007, 51:415-425

16. Michard F, Boussat S, Chemla D, Anguel N, Mercat A, Lecarpentier Y, Richard C, Pinsky MR, Teboul J-L: Relation between respiratory changes in arterial pulse pressure and fluid responsiveness in septic patients with acute circulatory failure. Am J Respir Crit Care Med 2000, 162:134-138.

17. De Backer D, Heenen S, Piagnerelli M, Koch M, Vincent JL: Pulse pressure variations to predict fluid responsiveness: influence of tidal volume. Intensive Care Med 2005, 31:517-523.

18. Girard TD, Bernard GR: Mechanical ventilation in ARDS: a state-of-the-art review. Chest 2007, 131:921-929.

19. Heenen S, De Backer D, Vincent J-L: How can the response to volume expansion in patients with spontaneous respiratory movements be predicted? Crit Care 2006, 10:R102.

20. de Backer D, Pinsky MR: Can one predict fluid responsiveness in spontaneously breathing patients? Intensive Care Med 2007, 33:1111-1117.

21. Soubrier $S$, Saulnier $F$, Hubert $H$, Delour $P$, Lenci $H$, Onimus $T$, Nseir S, Durocher A: Can dynamic indicators help the prediction of fluid responsiveness in spontaneously breathing critically ill patients? Intensive Care Med 2007, 33:1117-1124.

22. Garcia MIM, Cano AG, Monrové JC: Arterial pressure changes during the Valsalva maneuver to predict fluid responsiveness in spontaneously breathing patients. Intensive Care Med 2009, 35:77-84.

23. Zema MJ, Restivo B, Sos T, Sniderman KW, Kline S: Left ventricular dysfunction--bedside Valsalva manoeuvre. Br Heart J 1980, 44:560-569.

24. Lurie KG, Zielinski TM, Scott $H$, McKnite BS, Idris AH, Yannopoulos D, Raedler CM, Sigurdsson G, Benditt DG, Voelckel WG: Treatment of hypotension in pigs with an inspiratory impedance threshold device: a feasibility study. Crit Care Med 2004, 32:1555-1562

25. Yannopoulos D, McKnite S, Metzger A, Lurie KG: Intrathoracic pressure regulation improves 24 -hour survival in a porcine model of hypovolemic shock. Anesth Analg 2007, 104:157-162.

26. Westerdahl E, Lindmark B, Almgren SO, Tenling A: Chest physiotherapy after coronary artery bypass graft surgery - a comparison of three different deep breathing techniques. Rehabil Med 2001, 33:79-84. 